\title{
¿FUE LAKATOS UN REALISTA EPISTÉMICO?: EL ROL DE LA VERDAD EN LA METODOLOGÍA DE LOS PROGRAMAS DE INVESTIGACIÓN CIENTÍFICA ${ }^{1}$
}

Bruno Borge ${ }^{2}$

\begin{abstract}
Resumen: Un diagnóstico más que difundido acerca de la obra de Lakatos seńala que su proyecto soslayó por completo la cuestión de la verdad como parte central del análisis del conocimiento científico. En una línea semejante, Hacking afirma que Lakatos encontró en la metodología un sustituto para la verdad. Incluso quienes descreen de estas interpretaciones acuerdan respecto de que Lakatos falla en dar cuenta de la relación entre el desarrollo del conocimiento y el aumento de la verosimilitud. En el presente trabajo argumento que (a) el problema de la verdad es central al proyecto filosófico de Lakatos, y (b) es posible construir una interpretación alternativa en la que su proyecto de establecer un vínculo entre método y verdad pueda tenerse como exitoso. Propongo una lectura davidsoniana que adjudica a Lakatos un realismo epistémico.
\end{abstract}

Palabras clave: Imre Lakatos. Programas de Investigación Científica. Realismo Epistémico. Realismo Científico. Verdad.

\section{INTRODUCCIÓN}

Un diagnóstico más que difundido acerca de la obra de Lakatos señala que su proyecto soslayó por completo la cuestión de la verdad como parte central del análisis del conocimiento científico. Esta imagen, aunque popular, suele enmarcarse casi siempre en presentaciones informales. Tal vez la principal razón de ello es que los trabajos de Lakatos están plagados de referencias a la verdad o falsedad de ciertos enunciados científicos, y al hecho de que los

\footnotetext{
${ }^{1}$ Este trabajo fue pensado durante las clases y conversaciones que compartí con Ricardo Gómez en la Facultad de Filosofía y Letras de la Universidad de Buenos Aires en 2014. Sus comentarios a una versión preliminar resultaron de invaluable utilidad. Sean estas páginas un agradecimiento a su dedicación y calidad docente.
}

${ }^{2}$ Universidad de Buenos Aires / CONICET, Buenos Aires - Argentina. (D) https://orcid.org/00000002-1755-9690 E-mail: brunojborge@gmail.com

http://doi.org/10.1590/0101-3173.2020.v43esp.04.p47

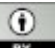

This is an open-access article distributed under the terms of the Creative Commons Attribution License. 
programas de investigación científica tienen mayor o menor contacto con una realidad que en buena medida determina su éxito, entre otras múltiples alusiones a tópicos conexos. Una versión más elaborada que goza de cierto prestigio - defendida por Hacking (1979) - no ignora esta situación, pero sostiene que las consideraciones lakatosianas respecto de la verdad (incluyendo algunas declaraciones explícitas en relación a su importancia) deben ser interpretadas en el contexto más amplio de un proyecto filosófico en el que, finalmente, acaban por ocupar un lugar marginal. Hay varios modos en que esta historia se ha contado, pero en todos algo (e.g. la metodología, la historia o una racionalidad pragmática) termina por empujar la verdad fuera de la escena. En cualquier caso, incluso quienes descreen de esta línea de interpretación suelen acordar respecto de que, aun siendo la cuestión de la verdad importante para el proyecto lakatosiano, su rol corresponde al de una pretensión fallida: brindar un relato adecuado acerca de cómo el desarrollo del conocimiento a lo largo de la historia de la ciencia - tal como lo describe la metodología de los programas de investigación científica - se vincula con una creciente aproximación a la verdad acerca del mundo. En otras palabras, que Lakatos no ha podido ofrecer una solución aceptable al problema de la relación entre método y verdad.

En el presente trabajo me propongo mostrar, en primer lugar, que la cuestión de la verdad es central al proyecto filosófico de Lakatos. Para ello ofrezco una reconstrucción de la interpretación de Hacking y de las razones pesimistas que se suelen alzar en contra del éxito de Lakatos en dar solución al problema de la relación entre método y verdad (sección 2). En la sección 3 ofrezco una fundamentación de mi afirmación respecto de la importancia de la verdad en la filosofía lakatosiana. El segundo objetivo de este trabajo consiste en construir una interpretación alternativa de Lakatos en la que su proyecto de establecer un vínculo entre método y verdad pueda tenerse como exitoso. En tal sentido, propongo en la sección 4 una lectura davidsoniana que adjudica a Lakatos la defensa de un realismo epistémico. Por último, la sección 5 incluye las conclusiones y comentarios finales a este trabajo.

\section{Arribando al PROBlema: la VERdAd y la METOdología de los PROGRAMAS DE INVESTIGACIÓN CIENTÍFICA}

Hacking (1979) ve en Lakatos a alguien empeñado es construir un nuevo acceso hacia la objetividad del conocimiento, luego de que Kant 
quemara los puentes de la teoría reprensentacional de la verdad. Su cruzada no sería otra que la que busca dar cuenta de la objetividad sin verdad, de la legitimidad objetiva del conocimiento científico por una vía distinta que la de la representación verdadera del mundo. De acuerdo con Hacking, el hemisferio hegeliano del corazón de Lakatos es el que inspira el camino a seguir: la verdad ha de ser reemplazada por el método. A la hora de evaluar un programa de investigación científica no ha de mirarse cuán adecuada es la imagen que ofrece acerca de los hechos a los que refiere o pretende referir, sino cuál es su contribución al crecimiento del acervo de conocimiento objetivo que la ciencia posee acerca de esos hechos. Mientras que lo primero requiere de nociones semánticas (referencia, verdad, verosimilitud etc.), lo segundo es determinable a partir de consideraciones meramente metodológicas. Lo importante en esta imagen del desarrollo del conocimiento no es tanto el conocimiento como el desarrollo, es decir, el aumento progresivo de nuestras descripciones del mundo a través de sucesivas adiciones e impugnaciones de pasos previos. El mismo Hacking se apresura a notar que, a diferencia de lo que ocurre en el sistema hegeliano, el éxito de esta empresa epistémica no está determinado por la dinámica inmanente a su desarrollo, sino depende de labor progresiva de la comunidad que la lleva a cabo. De ese modo, en la lectura de Hacking, el método adquiere una dimensión normativa que redobla su importancia, no solo es el reemplazo de la noción de verdad como valor nodal de la práctica científica, sino el foco donde ha de fijarse la atención para asegurar un desarrollo satisfactorio del conocimiento.

Esta interpretación, aunque profundamente influyente, no ha tenido una aceptación universal. Newton-Smith (2002, p. 101) reconoce los méritos de la lectura de Hacking, pero señala que "[...] no puede haber ninguna duda seria respecto de que para Lakatos la metodología de los programas de investigación científica es una ruta hacia la verdad"3. Con todo, sostiene que el proyecto lakatosiano es fallido, en tanto no consigue mostrar convincentemente una conexión entre la metodología de los programas de investigación científica y el objetivo de la ciencia (NEWTON-SMITH, 2002, p. 102). Un análisis crítico detallado de sus razones contra la interpretación de Hacking habrá de esperar hasta la sección siguiente, en cuanto a la vinculación entre la metodología y la verdad dentro del cuadro ofrecido por Lakatos la cuestión es mucho más compleja. Por su parte, Larvor (1998) sigue los pasos de esta crítica. Si bien no deja de reconocer que hay ciertas evidencias que

${ }^{3}$ A menos que se indique lo contrario, todas las traducciones me pertenecen. 
podrían apoyar la lectura de Hacking, concluye que una evaluación general que los elementos hegelianos en la filosofía de Lakatos están vinculados al carácter histórico de la metodología - y de la reflexión filosófica sobre ella más que a la noción de verdad.

Pese a estas voces adversas, la interpretación de Hacking conserva su vigencia en las lecturas contemporáneas de la obra de Lakatos y, en mi opinión, ejerce todavía su fuerza abonando la hipótesis (presente por ejemplo en las evaluaciones de Newton-Smith, Larvor y Smart) de que aun si la verdad fuera el objetivo final de la práctica científica y la metodología de los programas de investigación científica el medio para alcanzarlo, predomina en Lakatos un espíritu de resignación que hace transparente la imposibilidad de primera mano de conectar razonablemente método con verdad. Llamaré a esta tesis derrotista sobre la posición del propio Lakatos. Ha de reconocerse que no faltan en su obra pasajes que apoyan esta idea ${ }^{4}$, sin embargo, creo que han de considerarse dos cuestiones. En primer lugar, también puede encontrarse apoyo textual para una lectura más optimista respecto de la problemática de la verdad; y aun si así no fuere, o el balance entre ambas evidencias se juzgase favoreciendo aquel espíritu pesimista, eso no soslaya el hecho de que la aproximación a la verdad acerca del mundo natural ha sido para Lakatos un punto central en su concepción del conocimiento científico. En segundo término - y aún más importante - sostengo que la reelaboración de algunos elementos dentro del cuadro lakatosiano (muchos de ellos presentes en desarrollo de nociones basales de su filosofía, así como en las críticas que dirigió a propuestas alternativas) permiten brindar un panorama conceptual que favorece la mencionada lectura optimista respecto de la relación entre verdad y método.

Pero más allá del balance de ciertas declaraciones de carácter global que recorren la obra de Lakatos, hemos de prestar especial atención a aquellos aspectos conceptuales que han motivado lo que denomino tesis pesimista, según la cual la verdad es, o acaba siendo, un elemento marginal en la arquitectura de la epistemología lakatosiana. Si bien estas aproximaciones tienen múltiples asideros, considero que dos son los puntos centrales que la justifican:

(a) A pesar de rechazar rotundamente la importancia de los experimentos cruciales, la corroboración de predicciones noveles es el foco de los dictámenes

${ }^{4}$ Considérese, por ejemplo, el siguiente: "No existe una prueba última de que, incluso cuando las creencias Isabelinas hayan sido reemplazadas en el curso de cambios progresivos (como las creencias acerca del calor o el magnetismo), hayamos estado acercándonos hacia la Verdad. Solo podemos creer (no racionalmente), o más bien tener esperanzas de que aquello ha tenido lugar. A menos que la esperanza sea una 'solución', no hay solución al problema de Hume.” (LAKATOS, 1978b, p. 223). 
de progresividad de un programa, y por tanto la base del triunfo sobre sus rivales. Pero que ese sea el caso muestra que Lakatos, al igual que Popper, toma al aumento del contenido corroborado de una teoría como signo del aumento de su verosimilitud. Ahora bien, como el mismo Lakatos reconoce, solo la postulación de un principio inductivo extra-metodológico puede garantizar la correlación entre el éxito relativo de un programa y el aumento de su verosimilitud (1978a, p. 113). Pero, ¿en qué puede consistir ese principio? ¿Cómo puede considerárselo justificado? ¿Cómo sobreviviría a la devastadora argumentación popperiana contra la posibilidad de formular un principio tal? Al parecer Lakatos habría dejado estas y otras preguntas sin responder. Según Hacking (1979, p. 387), por ejemplo, "Lakatos no invirtió ningún esfuerzo en un principio inductivo, y sin embargo construyó algunas veces nociones bastante extrańas que servirían como un sustituto eficaz para una teoría de la verdad". Ello no sería trivial, en tanto la correlación necesaria entre el aumento del conocimiento y su grado de verosimilitud es la única vía para garantizar que el juego de la ciencia (expresión recurrente en la obra de Lakatos) no sea tan solo un juego.

(b) Lakatos ha defendido una suerte de visión retroactiva de la racionalidad, coherente con su recurrente rechazo a lo que llamaba racionalidad instantánea. No se trata meramente de cuestionar el papel de los experimentos cruciales en la elección de teorías, incluso la decisión de considerar progresivo o regresivo un programa de investigación corresponde a una instancia posterior al desarrollo de los hechos que la motivan. La racionalidad es más bien patrimonio de los epílogos, que de los prólogos, o las obras mismas. Esto tiene como consecuencia que el acuerdo de la teoría con la experiencia no es suficiente para garantizar su valor cognitivo, puesto que ello, en última instancia, ha de determinarse posteriormente.

El resto de este trabajo está dedicado a mostrar cómo el alcance de las tesis derrotista y pesimista puede ser relativizado, de modo tal que la cuestión de la verdad sea, además de un tema de especial importancia en la filosofía de Lakatos, una pieza conceptualmente vital para comprender su proyecto como una reconstrucción positiva del juego de la ciencia.

Antes, sin embargo, dedicaré algunas líneas a ubicar dicho proyecto en el marco de un debate algo más reciente que resulta de suma importancia para vislumbrar el sentido de mis objetivos: el debate acerca del realismo científico. 
Si bien este no es el lugar para hacer un desarrollo completo de la cuestión, baste decir que la tesis central del realismo científico afirma la existencia de las entidades inobservables postuladas por nuestras mejores teorías científicas. A esta tesis metafísica se suman una epistémica, que sostiene que es posible ganar un conocimiento (al menos aproximado) de dichas entidades, y una semántica, según la cual los enunciados que componen las teorías tienen valores de verdad (i.e., no son meros instrumentos de predicción). La posibilidad de afirmar, negar o mantenerse agnóstico respecto de cada una de estas tesis ha dado lugar a diversas posiciones que van desde la defensa del más duro realismo en las tres dimensiones de análisis, hasta variadas formas de antirrealismo5 .

¿Dónde, según las tesis derrotista y pesimista presentadas, deberíamos ubicar a Lakatos dentro de esta geografía? En lo concerniente a la dimensión metafísica, no se han alzado dudas de que Lakatos debe ser tenido por un realista. Por determinante que se juzgue la influencia hegeliana, la herencia del duro realismo popperiano ha dejado una huella indeleble en su obra. En cuanto a la dimensión semántica, todo indica, nuevamente, la afirmación de un realismo. Incluso si pudiesen ser relativizadas bajo consideraciones generales, el número de referencias a la verdad y falsedad de enunciados teóricos y observacionales es abrumador a lo largo de la obra de Lakatos.

En último lugar, resta la espinosa cuestión del realismo epistémico. Es claro que a la luz de las citadas tesis derrotista y pesimista Lakatos luce sosteniendo, al menos, un agnosticismo epistémico. El objetivo de este trabajo puede entonces reconstruirse como el intento de mostrar cómo la filosofía de Lakatos es compatible con un realismo epistémico.

Previo a fundamentar ese punto, sin embargo, es conveniente ahondar en un análisis que haga transparente el verdadero espíritu de la obra lakatosiana con respecto a la cuestión de la verdad, echando por tierra la tesis derrotista.

\section{LA VERDAD ACERCA DE LAKATOS Y LA VERDAD}

Según Hacking (1979, p. 384), “[...] el problema de Lakatos es proveer una teoría de la objetividad sin una teoría representacional de la verdad", puesto que "[...] puede encontrarse un reemplazo objetivo para la verdad en

\footnotetext{
5 Para un tratamiento pormenorizado de dichas variaciones véase Kukla (1998) y Borge (2015).

${ }^{6}$ Énfasis original. En las citas subsiguientes toda marca de énfasis debe leerse como perteneciendo al original, a menos que se indique expresamente lo contrario.
} 
la metodología" (HACKING, 1979, p. 386). Parte de las razones para este movimiento residen que "[...] el lado hegeliano de Lakatos da por sentada la imposibilidad de una teoría representacionalista de la verdad" (HACKING, 1979, p. 386).

De modo semejante, Smart (1989, p. 49-50) ha señalado que "el interés por la metodología surge del deseo de demarcar la ciencia de la nociencia y aclarar la naturaleza del razonamiento científico (tanto teórico como experimental) en ese contexto. A pesar de que la noción de verdad se incorpora a la discusión, resulta es de poca ayuda para las preocupaciones de la ontología”.

Como se ha visto, esta lectura que proyecta un Lakatos en última instancia despreocupado por la cuestión de la verdad ha recibido algunas críticas. Más allá de ellas, creo que pueden consignarse algunas razones adicionales que abonan su implausibilidad. En primer término, Hacking presupone que el aumento del conocimiento no solo es el punto de partida del análisis lakatosiano, sino también su objetivo último. Toda la metodología de los programas de investigación científica tiene por fin el desarrollo cuantitativamente progresivo del conocimiento y la demarcación entre desarrollos racionales e irracionales de ese conocimiento. Todo ello, sostiene, "está conducido por consideraciones internas acerca de la historia del conocimiento, y no depende de ninguna teoría de la verdad" (HACKING, 1979, p. 385). En mi opinión, esta interpretación se aparta radicalmente del sentido que Lakatos imprimió al proceso de evaluación de teorías, cuya característica más central es la prosecución del desarrollo cualitativamente progresivo del conocimiento (i.e., su constante mejoramiento) y no meramente su crecimiento. Ello queda claro en el siguiente pasaje. Luego de destacar los méritos de la teoría popperiana de la verosimilitud a la hora de definir en qué consiste el progreso a lo largo de una secuencia de teorías refutadas, Lakatos (1978a, p. 156) afirma:

Pero eso no es suficiente: tenemos que poder reconocer el progreso. Eso puede hacerse fácilmente con un principio inductivo que conecte la metafísica realista con la evaluación metodológica, la verosimilitud con la corroboración, que reinterprete las reglas del 'juego de la ciencia' como una teoría - conjetural - acerca de los signos del desarrollo del conocimiento, esto es, acerca de los signos de la creciente verosimilitud de nuestras teorias cientificas.

Esta iluminadora cita muestra a las claras que evaluación de teorías ha de centrarse el desarrollo cualitativo del conocimiento más que en su volumen. 
Para lo cual es necesario mucho más que consideraciones internas acerca de la historia del conocimiento: lo que se requiere no puede ser otra cosa que una teoría de la verdad.

En segundo lugar, ha de notarse que el desarrollo del conocimiento tiene lugar en aquel mundo-3 de las ideas que se han alienado del mundo físico y mental, legado popperiano que Lakatos admite sin reservas. Este hecho se ha invocado precisamente a favor de la interpretación en clave hegeliana, pero como ya se ha señalado, esa lectura ignora el hecho de que el sistema de Lakatos carece de las categorías dialécticas para asegurar su carácter autónomo y, lo que es más importante, necesariamente progresivo. La metodología de los programas de investigación científica viene a suplir esa falta, pero no podría cumplir dicha función sin la adición de un presupuesto metafísico que garantice que el método mueva los engranajes de la ciencia hacia su objetivo, a saber, la verdad. Esto se vincula estrechamente con la tercera de las razones que en las que sostengo la implausibilidad de la interpretación de Hacking: es con relación a esta cuestión (aunque esto se replica en variados aspectos de su obra) que Lakatos utiliza un concepto para nada hegeliano de verdad. Un concepto que parece hundir sus raíces en la tradición representacionalistacorrespondentista que según Hacking está rechazando.

Solamente tal 'principio inductivo' puede convertir a la ciencia de un mero juego, en un ejercicio epistemológico racional; de un conjunto de divertidas estratagemas escépticas llevadas a cabo por diversión intelectual, en la mucho más seria empresa falibilista de aproximarse a la Verdad acerca del Universo. (LAKATOS, 1978a, p. 113-114).

Si bien considero que estas razones son suficientes para desarticular la lectura radical de Hacking, debe tenerse presente que la tesis pesimista expuesta en la sección precedente está, como ya he adelantado, enmarcada en una concepción más amplia que encuentra en los trabajos de Lakatos un espíritu general de resignación respecto de que la metodología de los programas de investigación científica pueda dar cuenta de un acercamiento progresivo hacia la verdad. Refiriéndose justamente a la - a mi juicio, contundente - cita que acabo de reproducir, Hacking (1979, p. 386-387) agrega:

Creo que no es un accidente que [la cita corresponda a] un pasaje nervioso colocado entre paréntesis, en el que encontramos 'Verdad acerca del Universo' escrito con irónicas letras mayúsculas. [...] En otro lugar, [Lakatos] alienta a Popper a incorporar un 'soplo de inductivismo' que va a generar por lo menos una conjetura metafísica (inestable) acerca de 
la conexión entre el desarrollo del conocimiento y la aproximación a la verdad. Pero Lakatos parece haber visto muy claramente que no se llega a ninguna parte con la tontería popperiana acerca de la relevancia de la teoría de la verdad de Tarski para su proyecto, o con la subsecuente doctrina que llamó 'verosimilitud'.

La sorprendente atribución de un carácter irónico a la sentencia lakatosiana sobre la verdad acerca del universo, así como la denuncia de una certeza velada respecto de inviabilidad de su proyecto corresponden a un diagnóstico que (en un ligero contraste con las tesis pesimistas enunciadas) he llamado tesis derrotista: la idea de que Lakatos ha dejado en su obra los signos de sus escasas esperanzas acerca del éxito de su propuesta epistemológica. Avgelis (1989, p. 162-163) la expresa de este modo: "Lakatos es plenamente consciente de las graves dificultades que acompañan su enfoque de la evaluación de teorías".

Larvor (1998, p. 102) también deja traslucir esta idea cuando sentencia que "[e]sta falla [el fracaso en conectar método y verdad] está teñida por la sospecha de que Lakatos, a pesar de su retórica demarcacionista, está en realidad demasiado cerca de Kuhn y Feyerabend en los detalles de su modelo".

En una línea semejante, Smart (1989, p. 49) señala que

[... ni siquiera existe la menor garantía de que un programa de investigación progresivo no comience a degenerar repentinamente, y no hay garantía de que los programas de investigación progresivos nos guíen hacia la verdad. Es cierto que, en un momento dado, Lakatos le suplica a Popper "un soplo de inductivismo" en la forma de un principio inductivo sintético conjetural (Lakatos, 1978a, p. 159-67). Esto demuestra que Lakatos es consciente del problema. El inconveniente es que si el principio sintético es meramente conjetural, en realidad no nos da nada de la garantía que necesitamos.

Considero que hay, sin embargo, una serie de razones que echan por tierra esta hipótesis. En términos estrictamente descriptivos, ha de notarse que a lo largo de los dos volúmenes de los Philosophical Papers el problema de la relación entre el desarrollo del conocimiento y la aproximación a la verdad es abordado críticamente (es decir, no solo mencionado) en casi una veintena de ocasiones, una cifra algo elevada para quien lo considere un causa perdida, o el punto donde termina por naufragar toda su filosofía. Además, y fuera de 
consideraciones cuantitativas, el sentido positivo de su proyecto queda claro a lo largo de sus escritos.

Sostengo que la 'lógica del desarrollo del conocimiento' debe incluir además de la teoría lógico-metafísica de la verosimilitud de Popper - alguna teoría especulativa genuinamente epistemológica conectando los estándares científicos con la verosimilitud (LAKATOS, 1978a, p. 165).

Esta exigencia no consiste en una prescripción filosófica dirigida a uno, o algunos de los relatos posibles acerca de cómo se desarrolla el conocimiento científico. A la hora de evaluar teorías disponibles acerca de la racionalidad científica afirma que

[1]as únicas alternativas que nos quedan son las metodologias pragmáticas convencionalistas, coronadas con un principio global de inducción [...] El juego convencionalista consistiría en recolectar datos 'aceptables' y ordenarlos en los sistemas de encasillamiento [pigeonhole systems] más simples que sea posible. [...] Pero estos juegos cientificos carecen de toda relevancia epistemológica genuina a menos que se les superponga alguna clase de principio metafísico (o, si se quiere, 'inductivo') que diga que el juego, tal como es especificado por la metodología, nos dota de las mejores chances de aproximarnos a la Verdad. Tal principio torna entonces la pura convención del juego en conjeturas falibles, pero sin tal principio, el juego de la ciencia simplemente es como cualquier otro juego (LAKATOS, 1978a, p. 122).

El llamar la atención sobre una cita tan extensa obedece a más de una razón. Quisiera notar, en primer lugar, que la mayúscula en 'Verdad' no tiene ningún tinte de ironía, al menos hasta donde creo llegar a comprender las ironías. En segundo término - y, por supuesto, más importante -, Lakatos (luego de haber desestimado el justificacionismo) exige como un requisito esencial a toda forma de metodología pragmática convencionalista la adición de un principio que garantice que el juego de la ciencia tome contacto con la realidad. De ello depende, en última instancia, el éxito de cualquier proyecto epistemológico. En tal sentido, y en tercer lugar, ha de notarse que el mismo carácter falible de las conjeturas científicas deviene de la vigencia de tal principio; dicho de otro modo, la mera posibilidad de tomarnos en serio el proyecto de una racionalidad científica falibilista nace con la aceptación de aquel principio, de lo contrario no hay siquiera auténtica posibilidad de error sino simples convenciones vacías. Este punto no es menor si se considera 
que el falibilismo propio de las metodologías a las que Lakatos adhiere como única alternativa tiene su piedra de toque en la experiencia. El cuarto y último aspecto sobre el que quiero llamar la atención se relaciona con el anterior de un modo que espero se haga transparente a lo largo de estas páginas. Se trata en realidad de un detalle que me permito subrayar: Lakatos - en este al igual que en otros pasajes - destaca el hecho de que la solución al problema de la evaluación de teorías - pero, fundamentalmente, al problema de la evaluación de (meta)teorías de la racionalidad científica - es un principio de carácter metafísico. Su condición de principio inductivo es relegada a un segundo plano, e incluso presentada de un modo que sugiere que se trata de un rasgo accesorio. Las consideraciones precedentes, y en especial estas últimas observaciones son, a mi juicio, suficientes para desestimar la tesis derrotista. Queda claro que Lakatos presentó la exigencia de aquel principio metafísico como la base para la relevancia epistemológica de toda filosofía de la ciencia. Del otro lado solo queda el abismo del escepticismo radical que tanto se dedicó a combatir. Es preciso, sin dudas, no hacer caso omiso del hecho de que no contamos en los escritos de Lakatos con ninguna formulación explícita o justificación de dicho principio. Si bien no quisiera ensayar hipótesis especulativas respecto de esa circunstancia, me atrevo a señalar que la insistencia con la que se refirió a la cuestión es suficiente para no soslayar que la obra de Lakatos se vio tempranamente truncada por su prematura muerte.

Más allá de ello, las dos últimas observaciones acerca de la extensa cita de Lakatos decantan en las ideas que intento defender en la sección siguiente: ganar el juego de la ciencia requiere de dar cuenta de cómo el carácter progresivo de los programas de investigación científica constituye un progreso epistémico real, conduciéndonos a algún modo de realización de la Verdad usando la mayúscula lakatosiana en el que, sospecho, es su verdadero sentido: la correspondencia de una ciencia total con el mundo.

\section{Ganando el Juego de la Ciencia: La metodología de los programas de INVESTIGACIÓN CIENTÍFICA Y LA VEROSIMILITUD}

\subsection{UNIENDO VERDAD Y MÉTODO}

No hay dudas de que la metodología lakatosiana requiere trazar un puente entre el desarrollo del poder heurístico de los programas de investigación científica y el aumento de su verosimilitud, es decir, entre la dinámica del juego de la ciencia y su objetivo último. Como he tratado de 
mostrar, Lakatos era plenamente consciente de la importancia de esa tarea para todo su proyecto filosófico. Si bien juzgo exagerada la opinión de Hacking respecto de que Lakatos no invirtió ningún tipo de esfuerzo en fundamentar tal conexión (1979, p. 387) - juicio sin dudas teñido por su convicción de que la verdad es un valor prescindible en la filosofía de Lakatos - cierto es que los aportes positivos al desarrollo de dicho vínculo son escasos, y el carácter último de la solución termina por quedar en el terreno de la mera conjetura.

No obstante ello, algunas piezas permiten reconstruir mínimamente el cuadro. Como hemos visto, la teoría especulativa genuinamente epistemológica requerida es frecuentemente presentada como un principio de inducción, o principio cuasi-inductivo, sintético, que sea capaz de distinguir ciencia de pseudo-ciencia (LAKATOS, 1978a, p. 161). Asimismo, a la luz de ese principio, el desarrollo progresivo del juego de la ciencia, i.e., la corroboración del contenido empírico excedente de las nuevas teorías y el descubrimiento de fenómenos nuevos, puede ser leído como un signo de la verdad, despejando así la pura incertidumbre escéptica (LAKATOS, 1978a, p. 159 y 162). Su función, en última instancia, es explicar por qué jugamos el juego de la ciencia (LAKATOS, 1978a, p. 164). Se trata, entonces, de un principio sintético, falible, pero pasible de ser evaluado racionalmente (LAKATOS, 1978a, p. 163).

Frecuentemente los críticos insisten en señalar que Lakatos (1978b, p. 187) afirma que su principio inductivo puede ser "aceptado sin creer en él", a fin de mostrar lo endeble de sus esperanzas de solucionar el problema de la inducción. Esa apelación es sin embargo equívoca, ya que en Popper on demarcation and induction (capítulo 3 de Philosophical Papers Volume I), Lakatos sostiene que aquella afirmación - realizada en un texto previo, Changes in the problem of inductive logic (capítulo 8 de Philosophical Papers Volume II) - corresponde a una sección trivial en la que ofreció una solución insuficiente. Por el contrario, insiste Lakatos, una solución verdaderamente interesante tendría lugar solo si [el principio de inducción] fuese formulado de un modo suficientemente rico, como para, dicho de algún modo, permitirnos criticar nuestro juego científico desde su punto de vista (1978a, p. 164). El principio en cuestión tiene un carácter conjetural, pero ello no es muestra de una débil convicción o de la flaqueza de la solución que proporciona, sino del su condición de misma de ser un principio producto de la especulación metafísica, que encuentra su fundamentación en una evaluación puramente racional - aunque no meramente a priori, en tanto sus consecuencias respecto de la metodología científica le exigen a esta ciertos parámetros de éxito. 
Muchos son, como ha de notarse, los rasgos que caracterizan al principio metafísico de Lakatos (1978a, p. 165), con todo, no encontramos en su obra más que una formulación explicita de carácter provisorio: "[...] la metodología de los programas de investigación científica es más adecuada que cualquier otra metodología para aproximarnos a la verdad acerca de nuestro universo actual". A todas luces ello es menos un principio que una petición de principio, después de todo el punto crucial es en virtud de qué (i.e. ¡de qué principio!) la metodología lakatosiana se vincula con la verosimilitud ${ }^{7}$. Antes de ensayar mi propia respuesta, no quiera dejar de destacar un punto importante concerniente a la dimensión y alcance del problema que nos ocupa.

La metodología de los programas de investigación científica, tal como el falsacionismo popperiano o el confirmacionismo de Hempel, nos ofrecen un modo de caracterizar y evaluar metodológicamente la actividad científica, atado a ciertas convicciones filosóficas. En el caso particular de la metodología lakatosiana, contamos con criterios precisos acerca de las condiciones que un programa de investigación científica ha de reunir para ser considerado progresivo, como así también con estándares históricos de racionalidad que nos autorizan a juzgar la conveniencia de proseguir o abandonar la investigación de un programa ${ }^{8}$. Es posible considerarlos más o menos concluyentes, o incluso no acordar con ellos, pero ello no significa que no constituyan criterios en sí mismos. Pero podemos preguntarnos, ¿cómo nos las arreglamos para juzgar los criterios mismos? La respuesta de Lakatos es bien conocida: la historia de la ciencia nos brinda la base para determinar qué metodología resulta más progresiva: a partir de un repertorio de casos intuitivamente salientes de la historia de la ciencia podemos determinar qué metodología es capaz de reconstruirlos con la mayor justeza.

\footnotetext{
${ }^{7}$ Es interesante notar que el tratamiento de este punto en el curso de la evolución del debate sobre el realismo científico tomó un rumbo que, si bien es esencialmente diferente al propuesto por Lakatos, guarda no obstante ciertas similitudes. Lakatos proponía cerrar la brecha entre el éxito de una (su) metodología y la verdad apelando a un principio de inducción o conjetura metafísica. A finales de la década del 70 [a partir de la sugerencia de Putnam (1975)], comenzó a haber un consenso general respecto de que el vínculo entre éxito predictivo y verdad debía ser justificado mediante otra forma de razonamiento ampliativo, la llamada inferencia a la mejor explicación, aplicada en el nivel metacientífico: la mejor explicación del éxito de la ciencia es su verdad aproximada (LIPTON 1991, cap. 4). Más recientemente se ha hecho notar que esta estrategia para justificar el realismo fue acompañada por un creciente interés por determinar cuáles son los compromisos metafísicos necesarios para dar cuenta de la actividad científica desde el realismo (PSILLOS 2019). Aunque este no es el lugar para establecer putos de contacto entre la posición de Lakatos y el derrotero del debate sobre el realismo científico, vale la pena tener en cuenta estas coincidencias de modo general.

${ }^{8}$ Volveré más adelante en esta sección sobre la cuestión puntual del carácter histórico o retroactivo de la racionalidad lakatosiana.
} 
Dicha salida no está exenta de problemas. Papineau (1989), por ejemplo, sostiene que la estrategia de apelar a la historia de la ciencia como tribunal para juzgar metodologías cae en un dilema fatal. Si aceptamos sin más el catálogo inicial de sucesos científicos salientes, presuponemos una cierta noción de ciencia que hace a la evaluación toda caer en una flagrante circularidad. Si por el contrario, y tal como propone Lakatos, desandamos el camino revisando cuidadosamente el listado inicial a fin de determinar si las intuiciones iniciales corresponden a episodios propios de la historia interna, no influenciados causalmente de modo relevante por eventos correspondientes a la historia externa, entonces, en opinión de Papineau, debemos comprometernos con el hecho de que los seres humanos son perfectamente racionales cuando se encuentran libres de influencias externas (1989, p. 438).

Según entiendo, Papineau está sobresimplificando postura de Lakatos tanto acerca de la evaluación de metodologías como - y principalmente acerca de la racionalidad y la consecuente distinción entre historia interna y externa. En cualquier caso, está en lo correcto al afirmar que a menos que la cuestión del vínculo entre verdad y método quede esclarecida, el éxito de una metodología no es suficiente para evaluarla positivamente o preferirla sobre otras alternativas, puesto que, después de todo, el objetivo de la ciencia no es otro que la verdad. De acuerdo con el análisis de Papineau, este rasgo no es más que la herencia popperiana plasmada en la noción de aceptabilidad: al aceptar una proposición sin creerla evadimos toda cuestión relativa a la verdad y, de ese modo, pasamos por alto el problema de la inducción. Pero el precio a pagar es renunciar al realismo epistémico y no tener defensas contra las consecuencias relativistas de objeciones como las de Kuhn y Feyerabend (PAPINEAU, 1989, p. 434). Sin embargo, espero que lo afirmado hasta aquí y (fundamentalmente) el resto de esta sección sean suficientes para mostrar que ese diagnóstico no es adecuado en el caso de Lakatos. En primer lugar, por los esfuerzos de Lakatos por resaltar la necesidad de buscar un vínculo metafísico entre método y verdad; en segundo término, porque, sostengo, es posible encontrar dentro del terreno demarcado por el propio Lakatos un camino fructífero hacia el realismo epistémico que Papineau reclama.

Comencemos entonces con el verdadero trabajo. Mi sugerencia es la siguiente: puede que los críticos no hayan estado tan equivocados al señalar que siendo deudor de cierta herencia post-kantiana Lakatos puede haber aborrecido toda noción representacional de la verdad, junto con la suposición de que nuestras creencias han de evaluarse ante un tribunal constituido por 
hechos puros. Después de todo, muchos pasajes sugieren que su rechazo de lo que Popper llamó psicologismo se vinculó estrechamente con su convicción de que las proposiciones no han de fundamentarse sobre la base de sensaciones u observaciones puras, sino siempre en otras proposiciones. Todo esto, sin embargo, no significa que Lakatos tenga que renunciar al carácter objetivo de la elección de teorías, ni tampoco a la afirmación de que el conocimiento proporcionado por nuestros mejores programas de investigación se corresponde sustancialmente con la realidad. De hecho, como hemos visto, los aspectos convencionalistas y anti-representacionalistas de la obra de Lakatos conviven con una irrenunciable convicción acerca del carácter objetivo y (al menos aproximadamente) verdadero del conocimiento. Más precisamente, con el hecho de que el desarrollo del cuerpo del conocimiento, plasmado en la progresividad de los programas de investigación dominantes, da cuenta de una aproximación a la Verdad acerca del universo. Según entiendo, esa aparente tensión presente en los escritos de Lakatos puede resolverse apelando a una solución de corte davidsoniano: renunciar a la noción representacionalista de la verdad no es renunciar a la verdad, ni siquiera a la correspondencia, en tanto puede mostrarse que la coherencia del sistema de creencias científicas, sumada a una particular comprensión del origen del vocabulario observacional, pueden dar como resultado la correspondencia del conocimiento científico con la realidad objetiva que intenta esclarecer. Así es como voy a proceder: en primer lugar expondré muy brevemente los lineamientos generales de la posición de Davidson que considero centrales para la propuesta aquí ensayada, en segundo término, procuraré consignar los cuatro aspectos más relevantes de esa tesis para, por último, dar cuenta de cómo dichos aspectos pueden ser reconstruidos dentro de la filosofía lakatosiana. Esto no presupone, obviamente, que la posición de Davidson sea correcta, sino que es posible emprender en esa clave una lectura de la filosofía de Lakatos.

El lema davidsoniano de que la coherencia genera correspondencia encaja en una particular arquitectura conceptual que incluye, entre muchos otros elementos, una interpretación de la teoría de la verdad de Tarski, la convicción de que la verdad ha de ser tomada como un concepto primitivo, una teoría específica acerca del significado y la adquisición del lenguaje, y una caracterización causal de la relación entre el mundo y las creencias. La breve exposición que sigue soslayará muchos elementos importantes, amparada en la brevedad que la transición a mis conclusiones exige. 
Según Davidson, nuestras creencias están causalmente vinculadas con el mundo; tenemos las creencias que tenemos porque habitamos el mundo que habitamos (2001, p. 138). Eso explica que, al menos hasta cierto punto, hablantes competentes comparten un buen número de coincidencias de sentido común. Estoo que se vincula, a su vez, con el hecho de que los términos de un lenguaje son aprendidos e interpretados a la luz de las actitudes lingüísticas de nuestros semejantes ante uno y el mismo mundo que compartimos. Los significados de dichos términos no están en la cabeza, sino en el mundo, y su origen está causalmente determinado por ese mundo (DAVIDSON, 2001, p. 43-44). Con todo, nuestras creencias no se contrastan con hechos o sensaciones de las cuales son representación, no hay en el mundo que las justifique. Las creencias, en tanto contenidos proposicionales, se evalúan a partir de otras creencias (DAVIDSON, 1990, p. 303). Sin embargo, por la naturaleza de su origen, el conjunto de nuestras creencias tiende a ser mayoritariamente verdadero, puesto que aquello que las causa es, en general, aquello que las hace verdaderas - la falsedad, de hecho, tiene para Davidson sentido solo sobre un trasfondo de verdad. Eso no significa, por supuesto, que no podamos tener creencias falsas acerca del mundo, sino que el contenido falso del cuerpo de creencias tiende a ser depurado cuando dichas creencias se contrastan con el resto (2001, p. 153). En otras palabras, Davidson desestima la posibilidad de un error sistemático, tan frecuentemente invocada en argumentos escépticos. Es en ese sentido en que la coherencia asegura la correspondencia con la realidad objetiva, aun cuando, dado un enunciado particular, no tenga pleno sentido decir que es verdadero en virtud de representar adecuadamente ciertos hechos.

Esta apretada síntesis nos permite rescatar cuatro aspectos esenciales de esta imagen acerca de la referencia, el significado y la verdad.

1. Existe un único mundo objetivo, independiente de nuestras mentes. Nuestras creencias tienen su origen causal en ese mundo, y son verdaderas o falsas dependiendo de su contenido y de cómo es el mundo.

2. No existe algo así como un catálogo de hechos puros con los que contrastar nuestras creencias, el único modo de determinar su verdad es comparándolas con otras creencias. 
3. Nuestras creencias más básicas acerca del mundo tienden a ser naturalmente verdaderas en razón de su origen causal, no obstante, ese origen causal no juega un papel en su justificación.

4. La coherencia de nuestro cuerpo de creencias es garantía de su correspondencia con el mundo (independiente de la mente), en el que tienen su origen causal. Es por ello que somos capaces de tener conocimiento acerca de ese mundo.

Comencemos con estos aspectos en orden. El primero de ellos implica, por una parte, la adopción de un realismo metafísico, cuya ubicación en el mapa lakatosiano puede encontrarse sin dificultad. Pero, por otra parte, contiene la afirmación de que (i) existe un vínculo causal entre las creencias y el mundo, y del hecho de que (ii) es en relación a ese mundo que nuestras creencias resultan ser verdaderas o falsas. Ambos elementos, sostengo, pueden ser encontrados en la obra de Lakatos. Identificar (ii) requiere de hacer una distinción cuidadosa entre verdad y justificación. Como veremos a continuación, Lakatos no supone que sea el mundo mismo - o su reflejo en datos sensoriales, estimulaciones de las terminales nerviosas o enunciados protocolarios - el que justifica la determinación del valor de verdad de un enunciado. Por el contrario, son sus relaciones con otros enunciados los que posibilitan su verificación, sin embargo, eso no significa que si alcanzásemos un conocimiento objetivo acerca del mundo, ese conocimiento fuese acerca de otros enunciados. Algunos de los pasajes citados a lo largo de este trabajo ilustran que, de poder trazarse un vínculo entre método y verdad, tendríamos la garantía de poseer conocimiento (aproximadamente verdadero) acerca del mundo. La afirmación (i), en cambio, debe ser rastreada con más cuidado. Lakatos no se ocupó demasiado de cuestiones relacionadas con el significado de los términos, por lo que hay pocos indicios en su obra acerca de cómo es que los adquirimos. Por otra parte, su preocupación por el conocimiento objetivo relegó al origen de nuestras creencias acerca del mundo al estatus de una cuestión propia de la psicología. Pero es tal vez en este último punto donde podamos encontrar un camino promisorio para hacer plausible la interpretación que propongo. En repetidas ocasiones Lakatos expresó su adhesión a la ontología popperiana que distingue tres mundos o estratos de realidad, siendo, como es bien sabido, el mundo-1 el mundo material, el mundo-2 el de las creencias, deseos y estados psicológicos subjetivos, y el mundo-3 el correspondiente al conocimiento objetivo alienado. Es esta geografía parece adecuado afirmar 
que, así como el mundo-3 está causalmente determinado por el mundo-2, este último guarda la misma relación respecto del mundo material. Esta idea es coherente, además, con la insistencia de Lakatos en afirmar que son los hechos los que terminan por determinar la corrección de nuestras creencias, al tiempo que (como hemos visto) defiende la idea de que un enunciado solo puede ser justificado por otros enunciados.

Esta última idea hace patente la coincidencia del segundo aspecto de esta solución davidsoniana con lo expuesto por Lakatos. En variadísimas ocasiones se pronuncia contra la idea de que lo enunciados se justifiquen a partir de hechos, o sensaciones. Afirma, por ejemplo, que "ninguna proposición puede ser probada a partir de un experimento. Las proposiciones solo pueden ser derivadas de otras proposiciones, no pueden ser derivadas de los hechos: no pueden probarse enunciados a partir de experiencias." (LAKATOS, 1978a, p. $16)^{9}$. En tal sentido, no me detendré más en este punto.

El tercer aspecto considerado más arriba presenta un verdadero desafío. Debe quedar claro que Lakatos no ha hecho ninguna declaración explicita respecto del carácter naturalmente verídico de nuestras creencias, ni siquiera de la más básicas. Sin embargo, creo que la interpretación con la que pretendo reconstruir su posición es capaz dar hacer sentido esa afirmación. Mi principal punto a favor de esa posibilidad no es en realidad un argumento, sino la identificación de un marcado optimismo epistemológico por parte de Lakatos. Esa tendencia se hace patente, por empezar, en su defensa de una solución positiva al problema de la inducción, en su apuesta por la "débil luz" de dicha salida contra la "oscuridad total" del escepticismo: "sin ese principio las 'corroboraciones' o 'refutaciones' de Popper y mi 'progreso' o 'degeneración', se limitarían a simples títulos honoríficos en un mero juego" (LAKATOS, 1978a, p. 165). El mismo optimismo epistemológico cuya aparición tardía en la obra de Popper insistió en celebrar (LAKATOS, 1978a, p. 163; 1978b, p. 137). En ese contexto es que hay que comprender el especial estatus que gozan los enunciados que refieren a los aspectos observables del mundo: a pesar de no ser enunciados teóricamente neutros constituyen una clase especial de enunciados teóricos, capaces de mover los engranajes de la ciencia y de un modo no permeable a las objeciones relativistas, son el árbitro imparcial para las controversias cientificas (LAKATOS, 1978a, p. 46). Es en el marco de este optimismo epistemológico que hay que comprender la afirmación de

\footnotetext{
${ }^{9}$ Es claro que aquí, y mucho más en el contexto en que ocurre esta cita, que Lakatos utiliza 'derivar' en un sentido atinente a la justificación.
} 
que "son las 'verificaciones' más bien que las refutaciones las que proveen un punto de contacto con la realidad" (LAKATOS, 1978a, p. 51-52). El modo en que considero debemos ver este cuadro es el de un Lakatos que conserva la profunda convicción de que el progreso y la racionalidad científica se relacionan de algún modo con la aproximación a la verdad, siendo siempre la experiencia, nuestras creencias más básicas acerca de cómo es el mundo, las que nos brindan el punto de apoyo para impulsar ese movimiento. Sin ese punto de apoyo, sin ese contacto con la realidad, el direccionamiento del poder heurístico de los programas quedaría inexplicado. Este es el propósito del realismo, a partir del cual la afirmación de la verdad de nuestras creencias más básicas sobre el mundo puede hacerse inteligible. Esto revela, según creo, un aspecto sutil pero de profundo interés en la obra de Lakatos, a saber, su particular modo de resolver la tensión entre un inamovible falibilismo acerca del conocimiento, y la convicción de que el desarrollo de ese conocimiento tiene que estar de algún modo relacionado con el aumento de la verosimilitud. Volveré sobre este punto en el apartado siguiente.

El cuarto y último aspecto destacado en la solución dadvidsoniana que propongo - i.e. la coherencia genera correspondencia, por lo que somos capaces de tener conocimiento objetivo sobre el mundo - armoniza con todos los matices de la filosofía de Lakatos que he resaltado hasta ahora. Principalmente, con el hecho de que, si bien la experiencia no cumple ningún papel relevante en la justificación de las proposiciones científicas, el creciente grado de corroboración ha de ser leído como un signo de nuestra paulatina aproximación a la verdad. En tal sentido, Lakatos no solo se ha ocupado - como acabamos de ver de subrayar el valor de las "verificaciones" del contenido empírico de los programas, sino que ha reinterpretado el significado de las refutaciones para el cuerpo de conocimiento científico:

No se trata de que nosotros propongamos una teoría y la Naturaleza grite NO; más bien nosotros proponemos un entramado [maze] de teorías y la Naturaleza puede gritar INCONSISTENTE. Entonces el viejo problema de reemplazar una teoría refutada por los 'hechos' es reemplazado por el problema de cómo resolver inconsistencias entre teorías estrechamente asociadas. (LAKATOS, 1978a, p. 45).

Las refutaciones, es decir, la indicación de cierto contenido falso en nuestros programas, introducen inconsistencias en nuestro sistema de conocimiento. En una nota a pie de página Lakatos insiste en el hecho de 
que ese fenómeno no marca la refutación de una teoría en particular sino la necesidad de resolver la inconsistencia de manera progresiva (1978a, p. 45). Es precisamente el proceso de resolución de dichas inconsistencias el que motoriza el desarrollo de los programas de investigación en competencia, y por ende guía el conocimiento científico hacia su objetivo principal, i.e., la verdad. Como hemos visto, ese resultado no puede asociarse a la representación exitosa de la realidad por parte de las teorías en competencia, sino más bien al hecho de que la dinámica de desarrollo del conocimiento genera una aproximación a la verdad, en la medida en que la resolución de las inconsistencias determina la progresividad de los programas. En otras palabras, el restablecimiento de la coherencia ante sucesivas experiencias recalcitrantes da lugar a una dinámica que resulta en una aproximación paulatina a la verdad acerca del mundo.

La solución que he defendido ha de ser suficiente para echar por tierra los reparos impuestos por el punto (a) de la tesis pesimista presentada en la sección 2. No obstante, quisiera anticipar algunos posibles cuestionamientos. Podría seńalarse, por ejemplo, que la solución aquí presentada, en consonancia con la propuesta dadvidsoniana, apela a creencias individuales, mientras que Lakatos orienta su propuesta a un rango de conocimiento objetivo, carente de todo carácter psicológico. $\mathrm{Al}$ respecto basta con señalar que todas las alusiones a un cuerpo de creencias compartidas por una comunidad pueden cambiarse por referencias a un acervo de conocimiento objetivo, alienado de aquel mundo-2 al que las creencias pertenecen.

Una preocupación más seria puede surgir de considerar que aquello que estábamos buscando al comenzar esta sección, en relación con el punto (a), es la formulación de un principio de inducción, mientras que, a fin de cuentas, acabamos con piezas sueltas de una teoría del significado y de la verdad. Si bien responder a tal preocupación implicaría decir más de lo que este espacio permite, creo que basta con establecer dos puntos. En primer lugar, es trivialmente posible transformar lo dicho hasta aquí en un principio mediante el recurso elemental de poner en conjunción los enunciados elementales de la propuesta que he intentado esquematizar. Pero, en segundo lugar, creo que es vital destacar que en el marco del problema que nos ocupa Lakatos se ha referido alternativamente tanto a un principio de inducción, principio metafísico o teoría metafísica capaz de brindar una conexión entre método y verdad. De ese modo, la solución aquí propuesta podría corresponder más bien a la última de esas caracterizaciones. 
Por último, podría objetarse que la lectura que propongo salva el realismo epistémico de Lakatos a un precio muy alto, a saber, dar por sentada la corrección de la postura davidsoniana. Considero, sin embargo, que este camino crítico es estéril. Mi lectura, como hemos visto, no presupone que las posiciones de Davidson acerca de la verdad o justificación sean adecuadas, en el mismo sentido que la interpretación hegeliana de Hacking no requiere Hegel haya en efecto tenido razón respecto del desarrollo del conocimiento o la metodología. Más en general, una posición no necesita ser correcta para constituirse como una lectura realista (o antirrealista) respecto de la ciencia. El instrumentalismo y el realismo semántico abrazado por la mayoría de los realistas son posiciones contradictorias, por lo que, trivialmente, una de ellas es falsa. Sin embargo, ello no implica que debamos dejar de reconocer que ambas son, respectivamente, ejemplos de posiciones antirrealistas y realistas respecto del discurso científico. Si mi intento de revisitar el pensamiento de Lakatos a partir de categorías davidsonianas se juzga adecuado o iluminador, el realismo epistémico en Lakatos queda salvado independientemente de cuál sea nuestra opinión acerca de la corrección de la posición de Davidson.

\subsection{RACIONALIDAD Y VERDAD}

El punto (b) de la tesis pesimista considerada en la sección 2 destaca que la visión retroactiva de la racionalidad hace que el acuerdo de una teoría con la experiencia sea insuficiente para determinar el valor epistémico de una teoría. Dicho punto, creo, puede responderse de manera más sencilla. Después de todo, el hecho de que el verdadero significado de los eventos de la historia de la ciencia no pueda comprenderse sino después de pasado cierto tiempo no implica que una vez que esa comprensión ha sido alcanzada, una vez que el sentido propio de su racionalidad ha sido capturado, ese episodio en tanto tal no pueda tener un carácter progresivo que lo relaciones de un modo directo con la verdad. Puesto en otros términos, el hecho de que la corroboración o refutación de un conjunto de hipótesis pueda concretarse de modo instantáneo, si mediar un cierto proceso de recapitulación y compresión histórica, no es condición necesaria para que ese evento pueda considerarse como una contribución a la verosimilitud del conocimiento científico en general.

La preocupación respecto del carácter retroactivo de la racionalidad lakatosiana puede, sin embargo, darnos la posibilidad de evaluar un aspecto 
ya mencionado de su metodología que, según entiendo, resulta central para comprender la verdadera naturaleza de su proyecto. Me refiero a la tensión existente el arraigado falibilismo que recorre de punta a punta la filosofía de Lakatos y su optimismo epistemológico, o en términos más cercanos a la interpretación con la que intento reconstruir el pensamiento lakatosiano, la tensión que existe entre falibilismo y realismo epistémico. ¿Cómo es, después de todo, que pueden conciliarse el carácter perfectible y en algún sentido siempre provisorio de las hipótesis científicas con la convicción de que cada estadio del desarrollo del conocimiento es un paso que nos aproxima un poco más a la verdad acerca del universo? Sostengo que comprender el modo en que Lakatos resuelve esa tensión es vital para comprender no solo el sentido general de todo su proyecto y su concepción de la verdad sino, además, el verdadero carácter del realismo epistémico que intento adjudicarle. Puede que la mejor manera de elucidar este punto sea considerar las opiniones de Lakatos respecto de la cuestión de las leyes de la naturaleza, un punto pocas veces tenido en cuenta por los críticos a la hora de elaborar reconstrucciones de su pensamiento. Lakatos dedica un breve texto a clarificar las coincidencias y divergencias de las concepciones de Kneale y Popper respecto de ese punto, sentando luego las bases de su propia propuesta al respecto. El punto central de esa propuesta es la afirmación de que nuestros intentos por subsumir la vastedad del mundo natural bajo principios universales o leyes científicas encuentra una limitación en las posibilidades mismas de nuestro lenguaje. Las auténticas leyes de la naturaleza no podrían ser expresadas en enunciados finitos, por cuanto nuestro conocimiento sobre ellas puede ser positivo, puede ser acumulativo, pero ha de permanecer siempre como fragmentario. En tal sentido, se opone radicalmente a la afirmación de Popper respecto de que podríamos llegar a dar con una verdad última, aunque nunca lo sabríamos.

[Dicha tesis] contradice una de mis ideas preferidas aprendida del Marxismo (y no veo por qué debería resignarla). [...] Tal como Engels afirma explícitamente, la verdad final solo puede ser alcanzada 'desde un punto de vista práctico, por la sucesión sin fin de las generaciones humanas'. O, citando a Lenin: podemos 'acercarnos más más a la verdad objetiva (sin nunca llegar a alcanzarla)'. (LAKATOS, 1978b, p. 125). ${ }^{10}$

Es de ese modo que el rumbo de la ciencia hacia la verdad puede ser conciliado con el falibilismo: la aproximación asintótica hacia la verdad coincide con el carácter permanentemente falible del conocimiento, que, a su

${ }^{10}$ La cita original corresponde a Engels (1894, p. 122-23). 
vez, no invalida la posibilidad de comprender cada estadio particular del dicho conocimiento como un relato aproximadamente verdadero acerca del mundo natural. Uno puede o no coincidir con esta imagen de una verdad última que en sentido estricto no ha de efectivizarse nunca, más allá de ello, mi punto consiste en mostrar que en la resolución de la mencionada tensión reside el corazón del realismo epistémico de Lakatos ${ }^{11}$.

Es importante destacar, además, que, respecto de la fórmula popperiana, el rechazo de Lakatos es en realidad doble e involucra a ambos coyuntos. No solo rechaza la posibilidad de que, de hecho demos en un momento determinado con una verdad última, punto en el que ninguna predicción novel pueda ser formulada y ningún cambio pueda ser caracterizado como progresivo; Lakatos rechaza también la idea de que estando en contacto con algún rango de verdad acerca del mundo, esa circunstancia resulte en tanto tal epistémicamente inaccesible para nosotros. Puesto que la verdad se alcanza efectivamente en la práctica, ella se manifiesta en signos que pueden ser interpretados y comprendidos de modo tal que el desarrollo del conocimiento no sea otra cosa que el testimonio de nuestro acercamiento a la verdad.

\section{Conclusión}

En el curso de estas páginas procuré mostrar que la cuestión de la verdad es central para comprender el proyecto filosófico de Lakatos, cuyo sentido último dista mucho de ser el de una filosofía que pretende encontrar reemplazos para la noción de verdad. En eso consistió el rebatir las tesis derrotista y pesimista que podrían poner en duda ese punto. En el desarrollo de esa tarea brindé una interpretación que encuentra en Lakatos un realista no solo metafísico y semántico, sino también epistémico. En otras palabras, una lectura que, valiéndose de elementos presentes en la obra de Lakatos, pretende completar un cuadro en el que el proyecto de vincular el desarrollo del conocimiento con un aumento de la verosimilitud resulta exitoso. Creo que aun cuando de las tesis de este trabajo se juzguen inadecuadas, ellas son testimonio de la riqueza que el pensamiento de Imre Lakatos ofrece la reflexión epistemológica contemporánea. $\mathrm{O}$, dicho de otro modo, de que Lakatos es un clásico, lo que significa que todavía vale la pena volver a discutir con él.

${ }^{11}$ Una defensa completa de este punto puede encontrarse en Borge (2017). 
BORGE, B. Was lakatos an epistemic realist: the role of truth in the methodology of scientific research programs. Trans/form/ação, Marília, v. 43, p. 47-72, 2020. Edição Especial.

WAbstract: A widespread interpretation of Lakatos' work says his project completely sidestepped the question of truth as a central part of the analysis of scientific knowledge. In a similar vein, Hacking states that Lakatos found in methodology a substitute for truth. Even those who are skeptical of these interpretations agree that Lakatos failed to give an account of the relation between the growth of knowledge and increasing verisimilitude. In this paper I maintain that (a) the problem of truth is central to Lakatos' philosophical project, and (b) it is possible to develop an alternative interpretation in which his project to establish a link between method and truth can be seen as successful. I put forward a Davidsonian interpretation that ascribes to Lakatos an epistemic realism.

Keywords: Imre Lakatos. Scientific Research Programmes. Epistemic Realism. Scientific Realism. Truth.

\section{REFERENCIAS}

AVGELIS, N. Lakatos on the evaluation of scientific theories. En K. Gavroglu et al. (eds.), Imre Lakatos and Theories of Scientific Change. Kluwer: Dordrecht, 1989, p. $157-167$.

BORGE, B. Realismo Científico hoy: a 40 años de la formulación del Argumento del NoMilagro Acta Scientiarum. Human and Social Sciences v. 37 n.2, 2015, p. 221-233.

BORGE, B. Verdad y leyes de la naturaleza en la metodología de los programas de investigación científica. Signos Filosóficos. V. 37, n.1, 2017, p. 146-169.

DAVIDSON, D. The structure and content of truth. The journal of philosophy. V. 87, n. 6, 1990, p. 279-328.

DAVIDSON, D. Subjective, intersubjective, objective. Vol. 3. Oxford: Oxford University Press, 2001.

ENGELS, FRIEDRICH. Anti-Duhring: Herr Dühring's revolution in science. Foreign Languages Pub. House, 1894.

HACKING, IAN. Imre Lakatos's philosophy of science. British Journal for the Philosophy of Science. V. 30, n. 4, 1979, p. 381-402.

KUKLA, ANDRÉ. Studies in scientific realism. Oxford: Oxford University Press on Demand, 1998.

NEWTON-SMITH, WILLIAM. The rationality of science. Routledge, 2002.

LARVOR, BRENDAN. Lakatos: an introduction. Psychology Press, 1998. 
LAKATOS, IMRE, The methodology of scientific research programmes: Philosophical papers. Vol. I (J. Worrall \& G. Currie, Eds.), Cambridge: Cambridge University Press, 1978a.

LAKATOS, IMRE. Mathematics, science, and epistemology: Philosophical papers. Vol. $I I$ (J. Worrall \& G. Currie, Eds.). Cambridge University Press, Cambridge, 1978b.

LIPTON, PETER. Inference to the Best Explanation. London: Routledge, 1991.

PAPINEAU, DAVID. Has Popper Been a Good Thing? Imre Lakatos and theories of scientific change. Amstardam: Springer Netherlands, 1989, p. 431-440.

PSILLOS, STATHIS. El giro realista en la filosofía de la ciencia. En: B. Borge y N. Gentile (Eds.) La ciencia y el mundo inobservable. Discusiones contemporáneas en torno al realismo científico. Buenos Aires: Eudeba, 2019, p. 49-96.

PUTNAM, HILARY. Mathematics, Matter and Method. Vol. I. Cambridge University Press, Cambridge, 1975.

SMART, J. J. C. Methodology and ontology. En K. Gavroglu et al. (eds.). Imre Lakatos and Theories of Scientific Change. Dordrecht: Kluwer, 1989, p. 47- 57.

Recebido: 31/3/2017

Aceito: 11/11/2019 
BORGE, B. 